respectively, is equivalent to a transformation $T_{c}$ of the group, generated by the infinitesimal transformation

$$
c_{1} X_{1}+\cdots+c_{r} X_{r}
$$

with finite parameters $c_{1}, \cdots, c_{r}$; that is to say, if a system of finite values of the $c$ 's can be found to satisfy the symbolic equation $T_{b} T_{a}=T_{c}$. On page 282 we saw that the composition of the two arbitrary transformations $T_{a}$ and $T_{b}$ of the family defined by equations (4) was equivalent to a transformation $T_{0}$ of the family, with finite parameters $c$. But equations (4) were not in their canonical form, and therefore it did not necessarily follow that the transformation $T_{c}$ could be generated by an infinitesimal transformation of the group, as shown above. Consequently, if the finite equations of a group are not in their canonical form, the condition that for every finite system of values of the $a$ 's and $b$ 's a finite system of the $c$ 's can be found to satisfy the symbolic equation $T_{b} T_{a}=T_{c}$ is a necessary but not a sufficient condition for the continuity of the group.

University of Cincinnati, December, 1901.

\title{
SOME APPLICATIONS OF GREEN'S THEOREM IN ONE DIMENSION.
}

BY MR. OTTO DUNKKL.

(Read before the American Mathematical Society, February 22, 1902.)

Green's theorem ordinarily has reference to Laplace's equation in either two or three dimensions. It has been generalized however in the case of two dimensions by replacing Laplace's equation by the general homogeneous linear differential equation of the second order. In the generalized form the theorem relates not only to the given differential equation, but also to its adjoint differential equation.* A further extension of the theorem is possible by considering a differential equation of the $n$th order in two or more independent variables, and its corresponding adjoint. $\dagger$

* Cf. Encyklopädie, II, A. 7 c., p. 513.

$\dagger$ Cf. Darboux, Théorie des Surfaces, vol. 2, pp. 72, 74, for the case of two independent variables. 
It is desired here to consider the extension of the theorem to differential equations in only one independent variable, $i$. e., to the ordinary homogeneous linear differential equation of the second and higher orders, and to indicate very briefly some results which can be obtained from this extension, which we may call Green's theorem in one dimension.

We shall consider the general homogeneous linear differential expression

$$
P(y)=\frac{d^{n} y}{d x^{n}}+p_{1} \frac{d^{n-1} y}{d x^{n-1}}+\cdots+p_{n-1} \frac{d y}{d x}+p_{n} y,
$$

in which $p_{1}, p_{2}, \cdots, p_{n}$ are continuous real functions of the real independent variable $x$ in the interval $a \leqq x \leqq b$. We shall further assume that such of their derivatives as may be needed exist and are continuous.

If $y$ and $z$ are any two functions of $x$ with continuous derivatives of orders up to and including the $n$ th, then by integrating the formula of Lagrange,

$$
z P(y)-y \bar{P}(z)=\frac{d}{d x} P(y, z),
$$

where $\bar{P}(z)$ and $P(y, z)$ are respectively the adjoint and bilinear differential expressions of $P(y)$, we shall obtain the following integral formula, which we may designate as Green's theorem in one dimension,

$$
\begin{gathered}
\int_{a}^{b}[z P(y)-y \bar{P}(z)] d x=\left.P(y, z)\right|_{a} ^{b} \\
\left.P(y, z)\right|_{a} ^{b}=P[y(b), z(b)]-P[y(a), z(a)] .
\end{gathered}
$$

When the expression to which Green's theorem is applied is self-adjoint, $\dagger$ there is a second associated integral formula which here becomes

$$
\begin{aligned}
\int_{a}^{b} \sum_{i=0}^{m} & (-1)^{i} p_{m-i} y^{(i)} z^{(i)} d x \\
& =\int_{a}^{b} y P(z) d x-\left.\sum_{i=1}^{m} \sum_{j=0}^{i-1}(-1)^{j} y^{(j)} \frac{d^{i-j-1}}{d x^{i-j-1}}\left(p_{m-i} z^{(i)}\right)\right|_{a} ^{b} \\
& =\int_{a}^{b} z P(y) d x-\left.\sum_{i=1}^{m} \sum_{j=0}^{i-1}(-1)^{j} z^{(j)} \frac{d^{i-j-1}}{d x^{i-j-1}}\left(p_{m-i} y^{(i)}\right)\right|_{a} ^{b},
\end{aligned}
$$

\footnotetext{
* Cf. Schlesinger's Handbuch, vol. 1, pp. 53, 55, or Darboux, Théorie des surfaces, vol. 2, pp. 99, 100.

† Cf. Sohlesinger's H andbuch, vol. 1, p. 73; or Darboux, Théorie des su rfaces, vol. 2, p. 119, for characteristic form of self-adjoint expressions.
} 
where

$$
n=2 m, \quad y^{(i)}=\frac{d^{i} y}{d x^{i}}
$$

This formula is the analogon of the three term form of Green's theorem in the potential theory

$$
\begin{aligned}
\iint\left[\frac{\partial U}{\partial x} \frac{\partial V}{\partial x}+\frac{\partial U}{\partial y} \frac{\partial V}{\partial y}\right] d x d y & \\
& =-\iint U \Delta V d x d y-\int U \frac{\partial V}{\partial n} d s \\
& =-\iint V \Delta U d x d y-\int V \frac{\partial U}{\partial n} d s . *
\end{aligned}
$$

When $n=2$ the general differential expression

$$
P(y)=\frac{d^{2} y}{d x^{2}}+p \frac{d y}{d x}+q y
$$

can always be rendered self-adjoint by multiplying by the non-vanishing factor

$$
K=e^{\int_{a}^{x} p d x}
$$

and we can then write

$$
K P(y)=\frac{d K y^{\prime}}{d x}+G y
$$

where

$$
G=q K=q e^{\int_{a}^{x} p d x} .
$$

Applying Green's theorem to (6) we have

$$
\begin{aligned}
\int_{a}^{b} K[z P(y)-y P(z)] d x & =\left.K\left[z \frac{d y}{d x}-y \frac{d z}{d x}\right]\right|_{a} ^{b} \\
\int_{a}^{b}\left[K \frac{d y}{d x} \frac{d z}{d x}-G y z\right] d x & =\left.K y \frac{d z}{d x}\right|_{a} ^{b}-\int_{a}^{b} K y P(z) d x \\
& =\left.K z \frac{d y}{d x}\right|_{a} ^{b}-\int_{a}^{b} K z P(y) d x
\end{aligned}
$$

* Cf. Encyklopädie, II, A. 7 c., for the corresponding three term formula in the case of the general self-adjoint homogeneous partial differen tial equation of the second order. 
Special theorems may be obtained from these last two formulæ by making different suppositions as to $y$ and $z$. If $y$ and $z$ are solutions of $P(y)=0$ and $b=x$, we shall have from ( $\left.2^{\prime}\right)$ Abel's formula

$$
z \frac{d y}{d x}-y \frac{d z}{d x}=\frac{C}{K} \quad(C=\text { constant })
$$

If $y$ is a solution of

$$
\frac{d K_{1} y^{\prime}}{d x}+G_{1} y=0
$$

and $z$ is a solution of

$$
\frac{d K_{2} z^{\prime}}{d x}+G_{2} z=0
$$

we have from $\left(3^{\prime}\right)$

$$
\begin{aligned}
K_{1} z \frac{d y}{d x}-\left.K_{2} y \frac{d z}{d x}\right|_{a} ^{b}=\int_{a}^{b}\left(G_{2}-G_{1}\right) y z d x & \\
& -\int_{a}^{b}\left(K_{2}-K_{1}\right) \frac{d y}{d x} \frac{d z}{d x} d x
\end{aligned}
$$

the formula of Sturm;* and we may therefore regard Sturm's work as an application of Green's theorem in one dimension.

The following theorem results from $\left(2^{\prime}\right)$ :

If there exists a function $z$ of $x$ such that it and its first two derivatives are continuous in the interval $a \leqq x \leqq b$ and satisfy the relations

$$
\begin{gathered}
z>0, \\
P(z) \leqq 0,
\end{gathered}
$$

then $P(y)=0$ is a non-oscillatory differential equation in the interval $a \leqq x \leqq b . \dagger$

* Cf. Encyklopädie II, A. 7a, p. 442.

†Cf. a paper by Professor Bôcher, Bulletin, May, 1901, p. 333, for definition of non-oscillatory differential equation. In the same paper, p. 335, is a different proof of this theorem, and a number of special tests obtained from it. 
If we had used in Green's theorem $P(y)$ instead of the self-adjoint expression $K P(y)$, the relation (10) would be replaced by

$$
\bar{P}(z) \leqq 0 . *
$$

A theorem in regard to the non-homogeneous linear differential equation of the second order

$$
P(y)=r
$$

can be obtained, if we suppose $r$ is a function of $x$ that does not change sign in the interval $a \leqq x \leqq b$.

Suppose $y$ is a solution of (11) that vanishes together with its first derivative at $a$; and $z$ is a solution of the reduced equation

$$
P(z)=0
$$

that vanishes at $a$ and again at $c,(a<c \leqq b)$, but not between $a$ and $c$. Then the theorem is that $y$ will not vanish again in the interval $a<x \leqq c . \dagger$

The general Green's function in one dimension for equations of the $n$th order has been defined by Professor Bôcher. $\ddagger$ As an example of the use of such a function, we shall consider for simplicity only the equation of the second order (4). For a given point $\xi$ within the interval $a \leqq x \leqq b$, there is in general one and only one Green's function $G(x, \xi)$ of the first kind. Applying Green's theorem to $G(x, \xi)$ and any particular solution $y$ of (4), we have

$$
K(\xi) y(\xi)=-\left.K(x) y(x) \frac{\partial G(x, \xi)}{\partial x}\right|_{a} ^{b} .
$$

This corresponds to the integral formula which gives the value of a solution of Laplace's equation at any point within a closed curve by means of the values that the solution takes on along the curve.

For equations of higher order there are corresponding formulæ.

Harvard University, Cambridge, Mass.

February, 17, 1902.

\footnotetext{
* A theorem corresponding to this for the elliptic type of the homogeneous linear partial differential equation of the second order bas recently been given by Professor Bôcher in his lectures on partial differential equations.

$\dagger$ This theorem can also be proved by using the formula expressing the solution of (11) by means of a solution of the reduced equation.

$\ddagger$ Bulletin, A pril, 1901, p. 297.
} 